\title{
Functional analysis of the acetic acid resistance (aar) gene cluster in Acetobacter aceti strain 1023
}

\author{
Elwood A. Mullins, ${ }^{1,2}$ T. Joseph Kappock ${ }^{1}$ \\ ${ }^{1}$ Department of Biochemistry, Purdue University, West Lafayette, Indiana; \\ ${ }^{2}$ Department of Chemistry, Washington University in St. Louis, St. Louis, Missouri, USA
}

\begin{abstract}
Vinegar production requires acetic acid bacteria that produce, tolerate, and conserve high levels of acetic acid. When ethanol is depleted, aerobic acetate overoxidation to carbon dioxide ensues. The resulting diauxic growth pattern has two logarithmic growth phases, the first associated with ethanol oxidation and the second associated with acetate overoxidation. The vinegar factory isolate Acetobacter aceti strain 1023 has a long intermediate stationary phase that persists at elevated acetic acid levels. Strain 1023 conserves acetic acid despite possessing a complete set of citric acid cycle (CAC) enzymes, including succinyl-CoA:acetate CoA-transferase (SCACT), the product of the acetic acid resistance (aar) gene $\operatorname{aarC}$. In this study, cell growth and acid production were correlated with the functional expression of aar genes using reverse transcription-polymerase chain reaction, Western blotting, and enzyme activity assays. Citrate synthase (AarA) and
\end{abstract}

Correspondence: T. Joseph Kappock, Department of Biochemistry, Purdue University, 175 South University Street, West Lafayette, Indiana 47907-2063, USA. Tel. +1.765 .494 .8383 - Fax: +1.765 .494 .7897 .

E-mail: kappock@purdue.edu

Key words: AarA, SixA, AarC, citric acid cycle, acetate overoxidation.

Acknowledgments: the authors thank Dr. Koichi Kondo for providing the $A$. aceti strains used in this study, Kelly L. Sullivan for performing size-exclusion chromatography, and Kayleigh E. Nyffeler for performing PCR controls. This work was supported by the US National Science Foundation (MCB 0347250) and Purdue University Agricultural Research Programs.

Conference presentation: part of this paper was presented at the $3 r d$ International Conference on Acetic Acid Bacteria. Vinegar and Other products, 2012 Apr 17-20, Cordoba, Spain (http://www.uco.es/aab2012/).

Contributions: EAM acquired, analyzed, and interpreted data and drafted and revised the manuscript; TJK interpreted data and revised the manuscript.

Conflict of interests: the authors declare no potential conflict of interests.

Received for publication: 1 October 2012.

Revision received: 28 November 2012.

Accepted for publication: 3 December 2012

This work is licensed under a Creative Commons Attribution 3.0 License (by-nc 3.0).

@C Copyright E.A. Mullins and T.J. Kappock, 2013

Licensee PAGEPress, Italy

Acetic Acid Bacteria 2013; 2(s1):e3

doi:10.4081/aab.2013.s1.e3
SCACT (AarC) were abundant in A. aceti strain 1023 during both log phases, suggesting the transition to acetate overoxidation was not a simple consequence of CAC enzyme induction. A mutagenized derivative of strain 1023 lacking functional AarC readily oxidized ethanol but was unable to overoxidize acetate, indicating that the $\mathrm{CAC}$ is required for acetate overoxidation but not ethanol oxidation. The primary role of the aar genes in the metabolically streamlined industrial strain $A$. aceti 1023 appears to be to harvest energy via acetate overoxidation in otherwise depleted medium.

\section{Introduction}

The characteristic ability of acetic acid bacteria (AAB) to aerobically oxidize ethanol to acetic acid has been harnessed for millennia to produce vinegar. ${ }^{1}$ Traditional acetification methods often involve diauxic growth..$^{2-4}$ Cultures accumulate acetic acid in the first logarithmic growth phase (log phase) as ethanol is oxidized, maintain acetic acid during the first stationary phase, and then deplete acetic acid in the second log phase. Ideal strains for vinegar production exhibit little acetic acid consumption during the first log phase and an ability to tolerate high concentrations of acetic acid during a prolonged stationary phase. ${ }^{2}$ Modern industrial acetification methods avoid the second log phase and concomitant loss of acetic acid. ${ }^{5}$

A genetic screen of the industrial vinegar production strain Acetobacter aceti 1023 identified a cluster comprised of five genes that was essential for acetic acid resistance on solid media. ${ }^{6}$ Three genes in the aar gene cluster are required for acetic acid resistance: aarA encodes citrate synthase (CS), ${ }^{6,7}$ sixA encodes a phosphoprotein phosphatase (SixA) proposed to modulate citric acid cycle (CAC) enzyme synthesis, ${ }^{8}$ and $\operatorname{arC}$ encodes succinyl-CoA:acetate CoA-transferase (SCACT). AarA and AarC constitute part of a specialized, variant CAC that provides acetic acid resistance by catalyzing the overoxidation of cytoplasmic acetate to carbon dioxide (Figure 1). AarC facilitates this process by uncoupling catabolic activation of acetate from substratelevel phosphorylation and/or adenylation.

A. aceti strain 1023 lacks the glyoxylate shunt enzymes isocitrate lyase and malate synthase, which bypass the oxidative decarboxylations performed by CAC dehydrogenases, and succinyl-CoA synthetase, ${ }^{8}$ making flux through the CAC dependent upon AarC. In contrast, the type strain $A$. aceti NBRC 14818 possesses a complete CAC including succinyl-CoA synthetase, both glyoxylate shunt enzymes, and AarC (Figure 1). ${ }^{4}$

The feasibility of acetate assimilation as a means of rapidly reducing acetic acid levels in planktonic cultures has brought into question the relevance of the aar gene cluster for acetic acid resistance in liquid media. ${ }^{9}$ Acetate dissimilation, however, is compatible with an 
acetic acid resistance function. ${ }^{8}$ This study seeks to assess the importance of the aar products in liquid media and to correlate transcriptional and translational levels with acetic acid levels during diauxic growth.

\section{Materials and Methods}

\section{Materials}

Chemicals were purchased from Sigma-Aldrich (St. Louis, MO, USA) or Fisher (Houston, TX, USA) in the highest purity available. A. aceti strains were a generous gift from Dr. Koichi Kondo, Mizkan Group Corporation (Aichi, Handa, Japan) (Table 1) 6,10-12. Oligodeoxynucleotide (ODN) primers from Integrated DNA Technologies (Coralville, IA, USA) were used without further purification (Table 2). Restriction enzymes, DNA modifying enzymes, DNA polymerases, and DNA size standards were from New England Biolabs (Beverly, MA, USA). Horseradish peroxidase (HRP)-conjugated goat $\alpha$-rabbit $\operatorname{IgG}$ $(\mathrm{H}+\mathrm{L})$ and rabbit $\alpha$-chicken IgY $(\mathrm{H}+\mathrm{L})$ antibodies were from Jackson ImmunoResearch (West Grove, PA, USA) and Thermo Scientific (Waltham, MA, USA), respectively. A. aceti CS (AarA), AarA with a Cterminal hexahistidine affinity tag (AarAH6), and A. aceti SCACT (AarC) with a C-terminal hexahistidine affinity tag (AarCH6) were isolated as described previously., ${ }^{7,8}$ Polyclonal antibodies were generated in rabbits ( $\alpha$-AarCH6 and $\alpha$-SixAH6) or chickens ( $\alpha$-AarA) by Cocalico Biologicals (Reamstown, PA, USA) using pure recombinant proteins overproduced in $E$. coli C41(DE3).

\section{General analytical methods}

Absorbance measurements were recorded on an 8453 UV-visible spectrophotometer (Agilent Technologies, Santa Clara, CA, USA). Protein quantitation by the method of Bradford ${ }^{13}$ was performed using bovine serum albumin as a standard. Chemiluminescence was recorded using a Chemilmager 5500 imaging system (Alpha Innotech, San Leandro, CA, USA). DNA was sequenced by the staff of the Purdue University Genomics Core Facility. Protein mass was determined by matrix-assisted laser-desorption/ionization time-of-flight mass spectrometry (MALDI-TOF-MS) by the staff of the Purdue Proteomics Facility.

\section{Isolation of genomic DNA and sequencing of the aar gene cluster}

A. aceti genomic DNA (gDNA) was isolated using 20/G genomic tips (Qiagen, Valencia, CA, USA). Polymerase chain reaction (PCR) mixtures contained $1 \times$ Phusion HF buffer, $0.2 \mathrm{mM}$ dNTPs, $125 \mathrm{ng}$ gDNA, $0.2 \mu \mathrm{M}$ each of ODNs 2023/2053 (Table 2), and 1 unit Phusion DNA polymerase. Amplification was performed in three stages: stage 1 (1

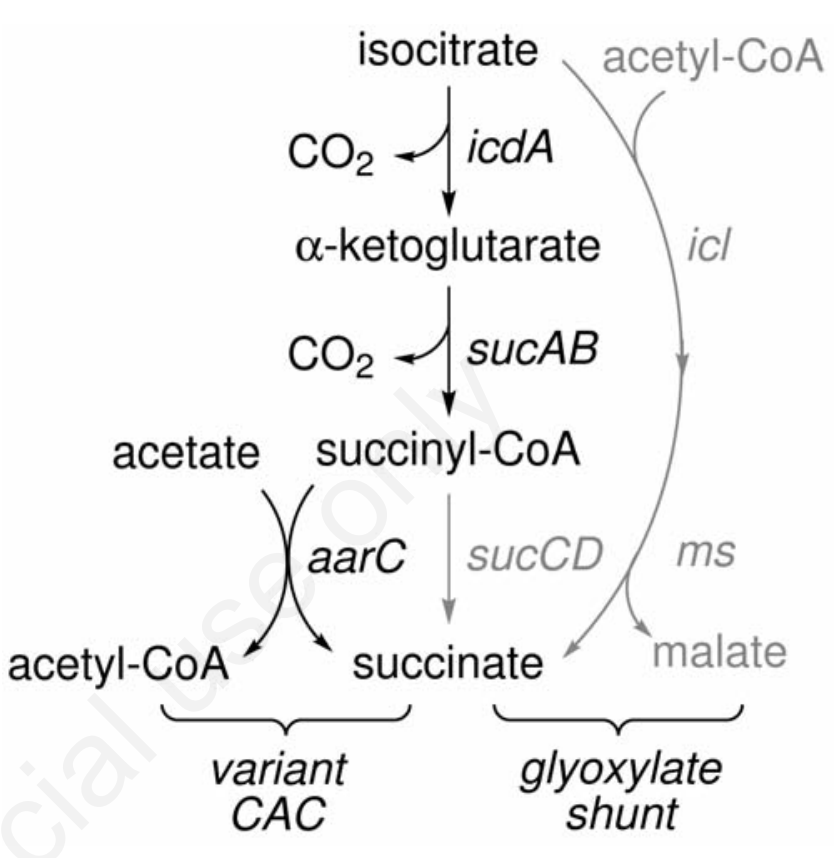

Figure 1. Citric acid cycle (CAC) variants and shunts found in diverse $A$. aceti strains. A portion of the canonical CAC is shown in the center. The AarC bypass and the glyoxylate shunt are shown to the left and right, respectively. Pathways absent in the vinegar production strain $A$. aceti 1023 are colored gray. All pathways are present in the type strain $A$. aceti NBRC 14818 .

Table 1. Strains and plasmids used in this study.

\begin{tabular}{|c|c|c|}
\hline Strain or plasmid & Description* & Source \\
\hline \multicolumn{3}{|l|}{ Strain } \\
\hline $\begin{array}{l}\text { A. aceti } \\
1023 \\
10-8^{\circ} \\
\operatorname{AS}^{\circ} 0^{\circ}\end{array}$ & $\begin{array}{l}\text { Vinegar factory isolate, } \text { Ace }^{\mathrm{R}} \\
\text { Derivative of strain } 1023, \text { pro }^{-} \text {Ace }^{\mathrm{R}} \\
\text { Derivative of strain 10-8, } \text { pro }^{-} \text {Ace }^{\mathrm{S}} \text { aarC }\end{array}$ & $\begin{array}{l}\text { Ref. } 10 \\
\text { Ref. } 11 \\
\text { Ref. } 6\end{array}$ \\
\hline $\begin{array}{l}\text { E. coli } \\
\text { C41(DE3) } \\
\text { DH5 } \alpha\end{array}$ & 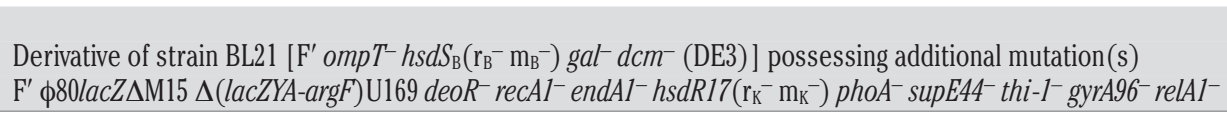 & $\begin{array}{l}\text { Avidis, Ref. } 12 \\
\text { Invitrogen }\end{array}$ \\
\hline \multicolumn{3}{|l|}{ Plasmid } \\
\hline pET23a & $\mathrm{T} 7$ promoter expression vector, $\mathrm{Ap}^{\mathrm{R}}$ & Novagen \\
\hline pJK385 & T7 promoter expression construct, encodes AarCH6, $\mathrm{Ap}^{\mathrm{R}}$ & Ref. 8 \\
\hline pJK502 & T7 promoter expression construct, encodes SixAH6, Ap ${ }^{\mathrm{R}}$ & Present work \\
\hline pJK504 & T7 promoter expression construct, encodes AarCH6-C357Y, Ap ${ }^{\mathrm{R}}$ & Present work \\
\hline
\end{tabular}

${ }^{*} \operatorname{aarC}$ deficient $\left(\operatorname{aar} \mathrm{C}^{-}\right)$, acetic acid resistant $\left(\mathrm{Ace}^{\mathrm{R}}\right)$, acetic acid sensitive $\left(\mathrm{Ace}^{\mathrm{S}}\right)$, ampicillin resistant $\left(\mathrm{Ap}^{\mathrm{R}}\right)$, and proline auxotrophic $\left(\right.$ pro $\left.^{-}\right)$; ${ }^{\circ}$ mutant $A$. aceti strains were created by chemical mutagenesis using $N$ methyl- $N^{\prime}$-nitro- $N$-nitrosoguanidine. ${ }^{6,11}$ 
cycle), 2 min at $98^{\circ} \mathrm{C}$; stage $2\left(30\right.$ cycles), $10 \mathrm{~s}$ at $98^{\circ} \mathrm{C}, 0.5 \mathrm{~min}$ at $65^{\circ} \mathrm{C}$, $2.5 \mathrm{~min}$ at $72^{\circ} \mathrm{C}$; and stage 3 ( 1 cycle), 5 min at $72^{\circ} \mathrm{C}$. PCR products were recovered using a kit (Qiagen) and sequenced using internal ODN primers. DNA sequences were assembled and edited using phred/ phrap/consed. ${ }^{14-16}$ Terminator sequences were identified using the ARNold web server. ${ }^{17}$

\section{Cloning and mutagenesis}

AarCH6-C357Y production plasmid pJK504 was prepared using the QuikChange II Site-Directed Mutagenesis kit (Stratagene, La Jolla, CA, USA), AarCH6 production plasmid pJK385, and ODNs 2028/2029 (Table 2). SixAH6 production plasmid pJK502 was prepared by cloning a sixA PCR product obtained using Vent DNA polymerase, A. aceti strain 1023 gDNA, and ODNs 2004/2005 (Table 2) into the NdeI and XhoI sites of plasmid pET23a (Novagen, Madison, WI, USA). ODN 2005 encodes a Ter175 $\rightarrow$ Ser mutation, which adds SLEHHHHHH to the C-terminus of SixA.

\section{Purification of SixAH6}

E. coli C41(DE3) cells freshly transformed with plasmid pJK502 were propagated on Luria-Bertani medium containing $10 \mathrm{~g} / \mathrm{L}$ tryptone, $5 \mathrm{~g} / \mathrm{L}$ yeast extract, $10 \mathrm{~g} / \mathrm{L}$ sodium chloride, and $0.1 \mathrm{~g} / \mathrm{L}$ ampicillin (LB/Amp) and supplemented with $0.2 \%(w / v)$ glucose. Production cultures (1 L) were inoculated with overnight cultures at a 1:100 dilution and grown at $37^{\circ} \mathrm{C}$ to an $\mathrm{OD}_{600}=0.6$. Cells were then harvested by centrifugation and resuspended in $1 \mathrm{~L} \mathrm{LB} / \mathrm{Amp}$ supplemented with $0.4 \mathrm{mM}$ isopropylthio- $\beta$ D-1-galactopyranoside (IPTG). Cultures were grown at $37^{\circ} \mathrm{C}$ an additional $3 \mathrm{~h}$. Cells were then pelleted by centrifugation and stored at $-80^{\circ} \mathrm{C}$. All subsequent steps were performed at $4^{\circ} \mathrm{C}$. Cells (typically $5 \mathrm{~g} / \mathrm{L}$ culture) were resuspended in $5 \mathrm{~mL} / \mathrm{g} 50 \mathrm{mM}$ potassium phosphate, $\mathrm{pH} 6.0$, and $100 \mathrm{mM}$ potassium chloride and disrupted by three cycles of sonication. Lysate was cleared by centrifugation at $30,000 \mathrm{~g}$ for $30 \mathrm{~min}$, addition of streptomycin to $1 \%(\mathrm{w} / \mathrm{v})$ from a $10 \%(\mathrm{w} / \mathrm{v})$ stock, incubation for $15 \mathrm{~min}$, and additional centrifugation at $30,000 \mathrm{~g}$ for $30 \mathrm{~min}$. Solid ammonium sulfate was then added to the cleared lysate to $25 \%$ saturation (144 g/L) over $30 \mathrm{~min}$. After equilibrating an additional $30 \mathrm{~min}$, solids were removed by centrifugation at $30,000 \mathrm{~g}$ for $10 \mathrm{~min}$. Solid ammonium sulfate was then added to the supernatant to $45 \%$ saturation ( $123 \mathrm{~g} / \mathrm{L}$ ) over $30 \mathrm{~min}$. After equilibrating an additional $30 \mathrm{~min}$, solids were collected by centrifugation at $30,000 \mathrm{~g}$ for $10 \mathrm{~min}$, dissolved in a minimal volume of TK buffer ( $50 \mathrm{mM}$ Tris $\bullet \mathrm{HCl}$, pH 8.0, and $100 \mathrm{mM}$ potassium chloride), and applied to a $\mathrm{Ni}^{2+}$-charged nitrilotriacetic acid (NiNTA) agarose column $(1.5 \times 4.5 \mathrm{~cm}, 8.0 \mathrm{~mL})$. The column was washed with 5 column volumes of TK buffer containing $20 \mathrm{mM}$ imidazole and then developed in a linear gradient of imidazole $(20 \rightarrow 500 \mathrm{mM}, 80 \times 80 \mathrm{~mL})$. Fractions containing SixAH6 were identified by sodium dodecyl sulfate polyacrylamide gel electrophoresis (SDS-PAGE), pooled, and concentrated to $>5 \mathrm{mg} / \mathrm{mL}$ by ultrafiltration (Amicon Ultra-15; 3000 MWCO). The concentration of imidazole was reduced by several cycles of dilution and reconcentration. Single-use aliquots were flash-frozen and stored at $-80^{\circ} \mathrm{C}$

\section{Size-exclusion chromatography}

Analytical size-exclusion chromatography was performed using an ÄKTA fast protein liquid chromatography system (Amersham Biosciences, Arlington Heights, IL, USA). SixAH6 ( $5 \mathrm{mg} / \mathrm{mL}$ in TK buffer adjusted to $5 \%$ glycerol) was filtered through a low-retention nylon membrane $(0.22 \mu \mathrm{m}$, Fisher) and then centrifuged at $16,000 \mathrm{~g}$ and $4^{\circ} \mathrm{C}$ for 10 min prior to injection $(0.1 \mathrm{~mL})$ onto a Superdex 200 column $(1.6 \times 60 \mathrm{~cm}, 120 \mathrm{~mL})$. The column was developed at $4^{\circ} \mathrm{C}$ and $1 \mathrm{~mL} / \mathrm{min}$ in TK buffer. Solution sizes were determined by reference to size standards (Sigma MW-GF-1000, Blue Dextran, and acetone) using $K_{a v}=\left(V_{e}-V_{0}\right) /\left(V_{t}-V_{0}\right)$, where $V_{e}$ is the peak elution volume, $V_{0}$ is the void volume, $V_{t}$ is the included volume of the gel bed, and $K_{a v}$ is the partition coefficient (proportional to the logarithm of the solution molecular weight).

\section{Heterologous overproduction of AarCH6-C357Y}

E. coli $\mathrm{C} 41$ (DE3) cells transformed with plasmid pJK504 were propagated on LB/Amp. Production cultures (1 L) were inoculated with overnight cultures at a 1:500 dilution and grown at $37^{\circ} \mathrm{C}$ to an $\mathrm{OD}_{600}=0.6$. Production of recombinant AarCH6-C357Y was then induced by addition of IPTG to $0.4 \mathrm{mM}$. Cells were grown at $15^{\circ} \mathrm{C}$ an additional $16 \mathrm{~h}$, harvested by centrifugation, and stored at $-80^{\circ} \mathrm{C}$. All subsequent steps were performed at $4^{\circ} \mathrm{C}$. Cells (typically $5 \mathrm{~g} / \mathrm{L}$ culture) were resuspended in $5 \mathrm{~mL} / \mathrm{g}$ TK buffer and disrupted by three cycles of sonication. Lysate was cleared by centrifugation at $30,000 \mathrm{~g}$ for $30 \mathrm{~min}$, addition of streptomycin to $1 \%(\mathrm{w} / \mathrm{v})$ from a $10 \%(\mathrm{w} / \mathrm{v})$ stock, incubation for 15 min, and additional centrifugation at $30,000 \mathrm{~g}$ for $30 \mathrm{~min}$.

\section{Growth of $A$. aceti cultures}

A. aceti strains 1023, 10-8, and AS10 (Table 1) were propagated on yeast extract-peptone-dextrose (YPD) medium containing $10 \mathrm{~g} / \mathrm{L}$ yeast extract, $20 \mathrm{~g} / \mathrm{L}$ peptone, and $20 \mathrm{~g} / \mathrm{L}$ dextrose and supplemented with $2 \%$ $(\mathrm{v} / \mathrm{v})$ ethanol (YPDE). YPDE solutions $(500 \mathrm{~mL})$ in $2.8 \mathrm{~L}$ Fernbach flasks were inoculated from single colonies, and cultures were grown at $30^{\circ} \mathrm{C}$ with continuous agitation (200 rpm) in a C25 incubator shaker (New Brunswick Scientific, Edison, NJ, USA) for $12 \mathrm{~d}$. Large aliquots $(10 \mathrm{~mL})$ were periodically removed, and cells were harvested by centrifugation. Cell-free medium samples and cell pellets were stored separately at $-80^{\circ} \mathrm{C}$ for subsequent analyses. Small aliquots $(3 \mathrm{~mL})$ were removed for RNA purification, mixed with 2 volumes of RNAprotect Bacteria reagent (Qiagen), and incubated at room temperature for 5 min. Cells were then harvested by centrifugation and stored at $-80^{\circ} \mathrm{C}$.

\section{Titration of acetic acid in $A$. aceti cultures}

Percent acidity in $A$. aceti cultures was determined by titration with $0.1 \mathrm{M}$ sodium hydroxide (standardized with potassium hydrogen phthalate) as described previously. ${ }^{3,18}$ Uninoculated YPD medium was titrated to determine the background percent acidity.

\section{Enzyme activity assays}

Frozen cell pellets (large aliquots) were resuspended in $10 \mathrm{~mL}$ icecold water, repelleted by centrifugation, and then resuspended in $1 \mathrm{~mL}$

Table 2. Oligodeoxynucleotides used in this study.

\begin{tabular}{lc} 
ODN & Sequence $\left(5^{\prime} \rightarrow 3^{\prime}\right)$ \\
413 & GAGCTCGAATTCTTAGCGTTTGGCAAGCGGCACATAGTCAC \\
416 & GATATACATATGAGCGCGTCGCAGAAAGAAGGTAAGCTATC \\
\hline 1221 & CAGGAAGCCCTCATATGACAGAGCG \\
1280 & GCAATACTCGAGTTATGATGGGAGC \\
\hline 2004 & GCCGCCATATGCGCCGCCTTGTTCTG \\
$2005 *$ & GGCATCTCGAGTGAGGGTCGCGCGAATGT \\
\hline 2023 & GCCGATACAACCACCAATAAAAGCT \\
$2028 *$ & GTTTAGGCTACATTGCCATGAACGGCATGA \\
\hline $2029 *$ & TCATGCCGTTCATGGCAATGTAGCCTAAAC \\
2037 & GCCGGCATATGACCGTGACAGACGACCA \\
\hline 2038 & GCCGGCATATGCGAACGGACTCCGCA \\
2053 & GGTAGCGTTATTCGCTCTGCCG \\
\hline${ }^{*}$ Changes in the coding region relative to the genomic sequence are underlined. oDN, oligodeoxynu-
\end{tabular}
cleotide. 


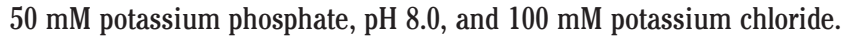
Cells were lysed by three cycles of sonication, and debris was removed by centrifugation $\left(16,100 \mathrm{~g}\right.$ and $4^{\circ} \mathrm{C}$ for $\left.15 \mathrm{~min}\right)$. SCACT and CS activities in soluble lysates were determined using 5,5 -dithiobis-(2-nitrobenzoic acid) dependent VisR and CS assays as described previously. ${ }^{8}$

\section{Isolation of RNA from $A$. aceti strain 1023}

RNA was isolated from cell pellets (small aliquots) according to the RNeasy Mini kit enzymatic lysis and proteinase K digestion protocol 4 (Qiagen). Contaminating gDNA was removed by double on-column DNase treatment using the RNase-Free DNase set (Qiagen). RNA used for transcriptional mapping was also subjected to in-solution DNase treatment and then repurified using the RNeasy Mini kit.

\section{Reverse transcription-polymerase chain reaction analysis}

One-step reverse transcription-polymerase chain reaction (RT-PCR) mixtures contained 1× Green GoTaq Flexi buffer, $4 \mathrm{mM}$ dithiothreitol, $1.25 \mathrm{mM}$ magnesium chloride, $0.2 \mathrm{mM}$ dNTPs, $1 \mu \mathrm{g}$ DNase-treated total RNA, $0.3 \mu \mathrm{M}$ of each DNA primer (Table 2: aarA, ODNs 413/416; sixA, ODNs 2004/2005; aarC, ODNs 1221/1280), 200 units Superscript II RNase $\mathrm{H}^{-}$reverse transcriptase (RT, Invitrogen, Grand Island, NY, USA), and 2.5 units GoTaq DNA polymerase (Promega, Madison, WI, USA). RT was omitted from control reactions. Amplification was performed in three stages: stage 1 ( 1 cycle), $30 \mathrm{~min}$ at $50^{\circ} \mathrm{C}, 5 \mathrm{~min}$ at $94^{\circ} \mathrm{C}$; stage 2 (20 cycles, aarA; 25 cycles, sixA and $\operatorname{aarC}$ ), $0.5 \mathrm{~min}$ at $94^{\circ} \mathrm{C}, 1$ min at $62.5^{\circ} \mathrm{C}, 1.5 \mathrm{~min}$ at $72^{\circ} \mathrm{C}$; and stage $3(1 \mathrm{cycle}), 5 \mathrm{~min}$ at $72^{\circ} \mathrm{C}$.

Transcript mapping was performed as described above except that the annealing temperature (stage 2) was reduced to $57.5^{\circ} \mathrm{C}$ and the number of cycles in stage 2 was increased to 30 . RT was omitted from additional control reactions lacking RNA but containing $10 \mathrm{ng}$ gDNA.

\section{Western blot analysis}

Soluble proteins present in cell-free lysates generated for $A$. aceti strain 1023 activity assays were separated by SDS-PAGE (12\%), and proteins were transferred to a polyvinylidene difluoride membrane $(0.2$ $\mu \mathrm{m}$, BioRad, Hercules, CA, USA) in Towbin buffer. ${ }^{19}$ [The AarC transfer buffer was supplemented with $0.1 \%(\mathrm{w} / \mathrm{v})$ sodium dodecyl sulfate (SDS).] The membrane was blocked for $1 \mathrm{~h}$ with TTBS buffer ${ }^{19}$ and probed for $1 \mathrm{~h}$ with one of three custom primary polyclonal antibodies (diluted 1:50,000 in TTBS). After rinsing with TTBS, the membrane was probed with one of two secondary HRP-conjugate antibodies $(\alpha$ rabbit, diluted 1:250,000 in TTBS; $\alpha$-chicken, diluted 1:500,000 in TTBS). The membrane was rinsed with TTBS and soaked for $5 \mathrm{~min}$ in a 1:1 mixture of stable peroxide and luminol/enhancer solutions (Thermo Scientific). Chemiluminescence was promptly measured using 1-5 min exposures.

\section{Data analysis and figure generation}

Image $\mathrm{J}^{20}$ was used to determine relative Western blot and RT-PCR band intensities. Molecular graphics images were created using
PyMOL..$^{21}$ Models of mutant proteins were prepared using the PyMOL mutagenesis wizard and the lowest-energy side chain rotamer conformation.

\section{Results}

\section{Overproduction, purification, and characterization of SixAH6}

The sixA gene region was originally annotated as $A a r B,{ }^{6}$ a protein with no known function and no known homologues. All attempts to heterologously overproduce AarB were plagued by apparent toxicity and failed to yield soluble protein (Susan C. Hockings, unpublished observations). Early attempts to clone sixA and overproduce SixAH6 were similarly hindered. However, soluble recombinant protein ( $\sim 50 \mathrm{mg}$ per L culture) was successfully produced using freshly transformed cells and growth medium supplemented with glucose to suppress leaky gene expression..$^{22}$ SixAH6 was purified by ammonium sulfate fractionation and immobilized metal affinity chromatography. While SDS-PAGE and Western blots showed multiple bands that cross-reacted with a polyclonal $\alpha$-SixAH6 antibody (Figure 2A,B), MALDI-TOF-MS indicated that SixAH6 was a homogeneous 183-residue protein retaining Met1 (Figure 2C). Size-exclusion chromatography suggested SixAH6 is primarily monomeric in solution (data not shown).

\section{Diauxic growth of $A$. aceti cultures}

A. aceti strains $1023,10-8$, and AS10 (Table 1) were propagated in YPD medium supplemented with $2 \%$ (v/v) ethanol. Both $A$. aceti strains 1023 and 10-8 exhibited biphasic growth (Figure 3A). The first log phase for all strains coincided with ethanol oxidation and acetic acid accumulation (Figure 3B). A. aceti strains 1023 and 10-8 maintained relatively constant levels of acetic acid throughout the first stationary phase but rapidly depleted levels of acetic acid as the second log phase began. In contrast, A. aceti strain AS10 remained in the first stationary phase and maintained a fixed level of acetic acid. Sequencing $(\geq 4.3$ fold coverage) of the $5.1 \mathrm{~kb}$ PCR amplicon containing the complete aar gene cluster revealed three missense mutations, encoding both AarC (strain AS10) and AarA (strains AS10 and 10-8) mutants (Table 3). ${ }^{23}$ Mutation of $\operatorname{aar} C$ may account for the Ace ${ }^{\mathrm{S}}$ phenotype of $A$. aceti strain AS10. An Ace ${ }^{\mathrm{R}}$ phenotype was conferred by plasmid pAR248, which contains the orf1 - aarC intergenic region and all of aarC (GenBank ${ }^{\mathrm{TM}}$ accession number DQ631551, starting at nucleotide 3141; Table 3). ${ }^{6}$

\section{Determination of citrate synthase and succinyl- CoA:acetate CoA-transferase activities}

Cells were harvested from $A$. aceti cultures during the $12 \mathrm{~d}$ growth period, and CS and SCACT activities were assayed in soluble lysates (Figure 3C). A. aceti strain 1023 exhibited moderate CS and SCACT activities in the first log phase and early first stationary phase, as

Table 3. Sequence differences in the aar gene clusters of $A$. aceti strains 1023, 10-8, and AS10.

\begin{tabular}{lccc} 
A. aceti strain & Mutation $(\mathrm{s})$ & Mutant(s) & GenBank accession number \\
1023 & None & None & DQ631551* \\
$10-8$ & $619 \mathrm{G} \rightarrow \mathrm{A}$ & AarA-A265V & JX475924 \\
\hline AS10 & $1139 \mathrm{C} \rightarrow \mathrm{T}$ & AarA-E92K & 5041 \\
& $4602 \mathrm{G} \rightarrow \mathrm{A}$ & AarC-C357Y & JX475925 \\
\hline
\end{tabular}

*The sequence was updated with an $\mathrm{A}$ at nucleotide position 1263 , not the $\mathrm{G}$ present in the original assembly. ${ }^{23}$ This correction is a silent alteration of the AarA Phe 50 codon. 
ethanol was being converted to acetic acid and glucose was presumably being used as the primary carbon source. CS and SCACT activities dropped dramatically ( $\sim 100$-fold decrease) during the first stationary phase and then spiked ( $>200$-fold increase) during the second log phase as acetic acid was depleted. In contrast, $A$. aceti strain 10-8 displayed very low CS and SCACT activities during both the first log and first stationary phases. Only upon onset of the second log phase did CS and SCACT activities rise to approximately those of $A$. aceti strain 1023. A. aceti strain AS10 exhibited CS and SCACT activities near the threshold of detection throughout the growth period.

\section{Translational analysis of AarA, SixA, and AarC}

Western blotting was performed to monitor AarA, SixA, and AarC levels in A. aceti strain 1023 (Figures $3 \mathrm{E}$ and 4). Congruent with activity assays, AarA and AarC levels were moderate in the first log phase, low in the mid first stationary phase, and highest in the second log phase

A

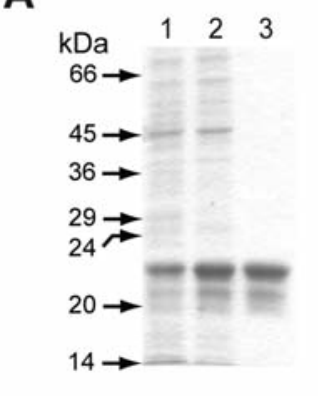

C

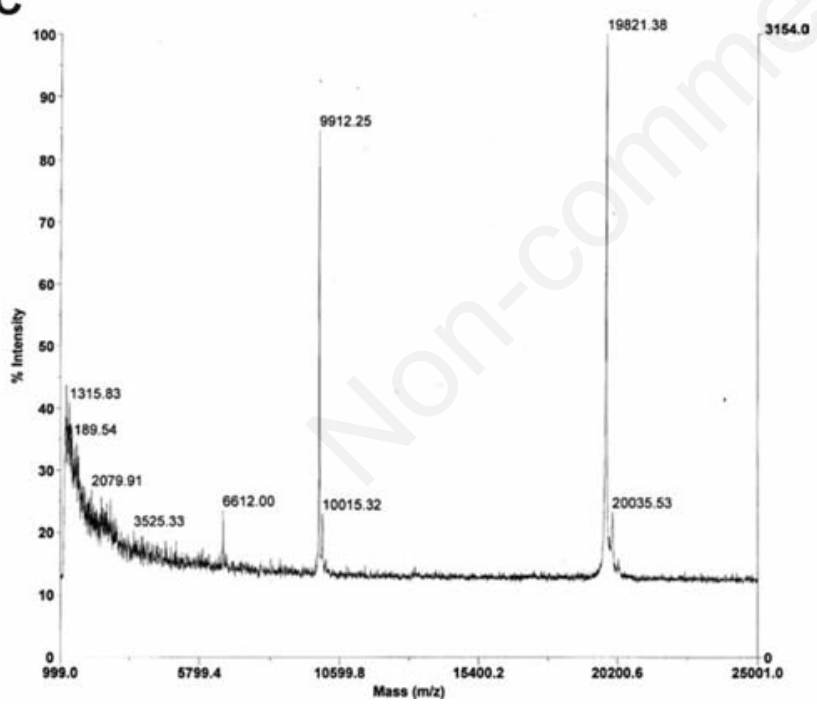

Figure 2. Isolation, analysis, and characterization of SixAH6. (A) Purification of SixAH6 monitored by SDS-polyacrylamide gel electrophoresis. Lane 1, clarified lysate; lane 2, 25-45\% ammonium sulfate fraction; lane 3, pooled NiNTA fractions. Pure SixAH6 migrates more slowly than expected and smears, consistent with incomplete denaturation or partial refolding during electrophoresis. Each lane contains $5 \mu \mathrm{g}$ protein. Size standard positions are indicated. (B) Semi-quantitative Western blotting of SixAH6 using an $\alpha$-SixAH6 antibody. Pure SixAH6 was added to $A$. aceti strain 1023 clarified lysate $(112 \mathrm{~h}$ point in Figure 3; $5 \mu \mathrm{g}$ total protein per lane) to give a two-fold serial dilution series. The limit of detection was $0.4 \mathrm{ng}$ SixAH6. (C) MALDI-TOF-MS analysis of SixAH6 $(19,821 \pm 2$ Da observed; 19,822 Da expected). as acetic acid was depleted from the medium. SixA was not detected at any stage of the growth period. Spiking of $A$. aceti strain 1023 lysate with pure SixAH6 (Figure 2B) was used to rule out problems with protein transfer to the blotting membrane and to establish an upper limit for SixA levels in the cell ( $\sim 500$ molecules).

\section{Transcriptional analysis of aarA and aarC}

Qualitative RT-PCR experiments were performed to monitor expression of aarA and aarC in A. aceti strain 1023 during growth in YPDE medium (Figures 3D and 5). Transcription of aarA and $a a r C$ increased

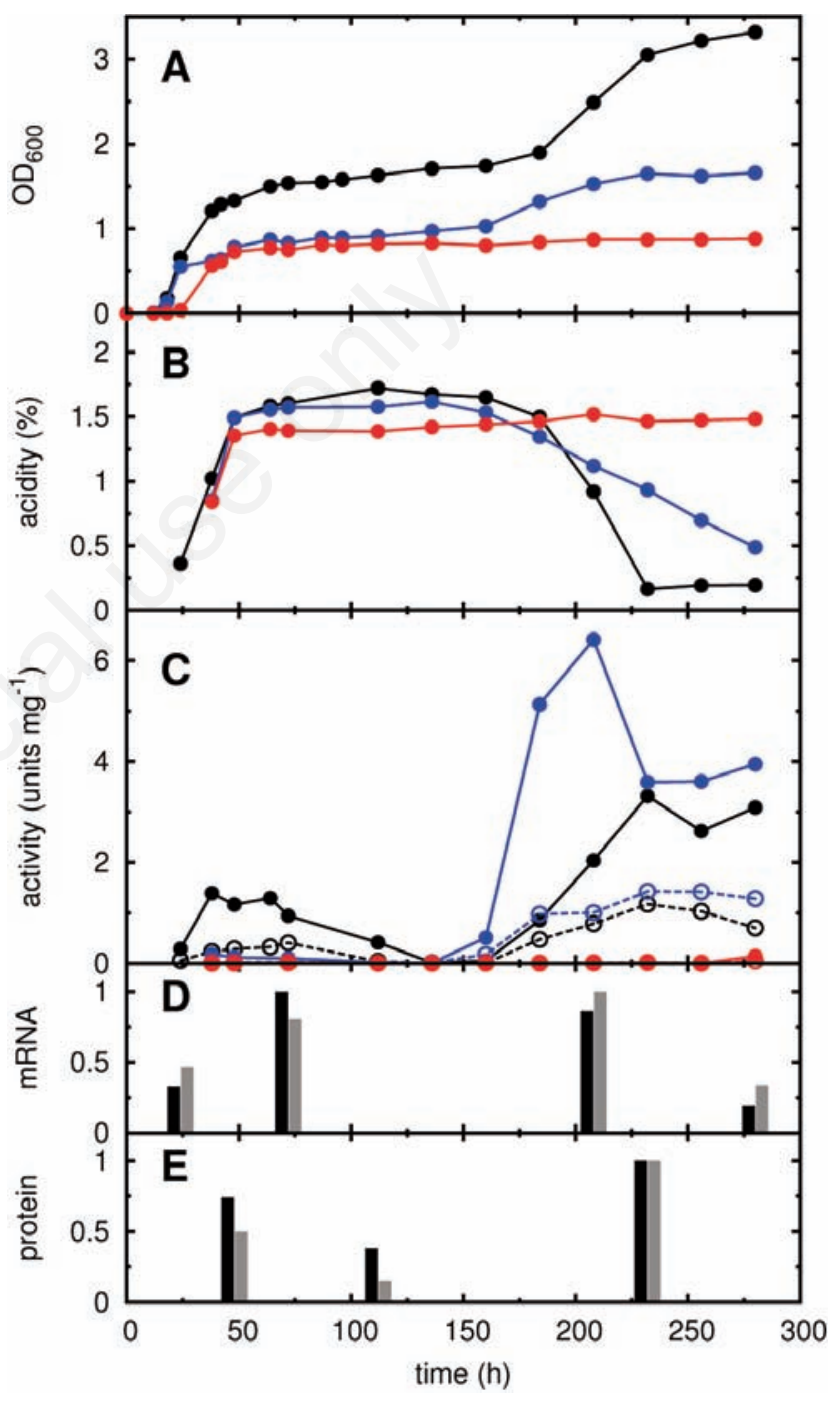

Figure 3. Propagation and analyses of $A$. aceti strains $1023\left(\right.$ Ace $^{\mathrm{R}}$, black symbols), 10-8 (pro- Ace ${ }^{\mathrm{R}}$, blue symbols), and AS10 (pro $\mathrm{Ace}^{\mathrm{s}}$ a arC $\mathrm{C}^{-}$, red symbols) in YPDE medium. (A) Cell density. (B) Titrable acidity. Complete conversion of $2 \%(\mathrm{v} / \mathrm{v})$ ethanol $(0.34$ $\mathrm{M})$ to acetic acid $(0.34 \mathrm{M})$ would correspond to $2.1 \%$ acidity. $(\mathrm{C})$ CS (AarA, solid lines) and SCACT (AarC, dashed lines) activities in soluble lysates. One unit is equivalent to one $\mu$ mol product formed per min. (D) Determination of aarA (black bars) and aarC (gray bars) levels in $A$. aceti strain 1023 by RT-PCR. Aliquots were taken at the time points indicated by the center of each pair of bars. Band intensities are given relative to the brightest band in Figure 5. (E) Determination of AarA (black bars) and AarC (gray bars) levels in $A$. aceti strain 1023 by semi-quantitative Western blotting. Aliquots were taken at the time points indicated by the center of each pair of bars. Band intensities are given relative to the darkest band (excluding lane C) in Figure 4. 
as acetic acid was produced in the first log and early first stationary phases. Transcription of $\operatorname{arC}$ further increased in the second log phase as acetic acid was consumed. The observed levels of expression correlate with AarA and AarC levels and CS and SCACT activities. In preliminary studies, transcription of $s i x A$ appeared to be greatest in the first log phase (data not shown).

\section{time (h)}

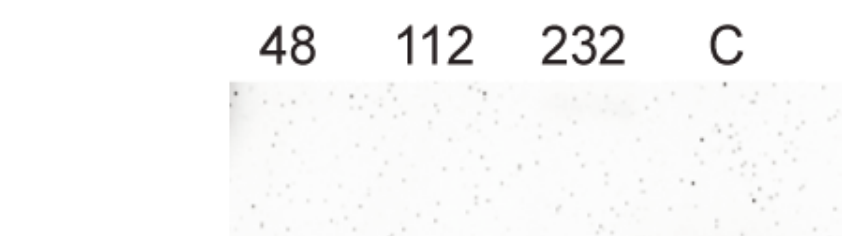

\section{AarA}

\section{AarC}

Figure 4. Analysis of AarA, SixA, and AarC by Western blotting. Lanes 48, 112, and 232 each contain $5 \mu \mathrm{g}$ total protein. Lane $C$ contains $50 \mathrm{ng}$ pure AarA, SixAH6, or AarCH6. SixA was not detected at any stage of the growth.

\section{Mapping of transcripts produced from the aar gene cluster}

The divergent orientation of $a \operatorname{ar} C$ relative to the other four genes in the aar gene cluster implies that $a a r C$ is expressed as a separate transcript. The number of transcripts produced by the remainder of the gene cluster was established by RT-PCR, using primer pairs that spanned the intergenic regions. RT-PCR products were observed for sixA - tyrA and sixA - orf1 but not for aarA - sixA (Figure 6A/B), suggesting that three aar transcripts are produced: a single polycistronic transcript including sixA, tyrA, and orf1 (RNA-B) and two monocistronic transcripts including aarA (RNA-A) and aarC (RNA-C) (Figure 6C). Transcription of RNA-A and RNA-C likely ends at the two rho-independent terminators that demarcate the aar gene cluster.

\section{In silico and in vitro characterization of AarA and AarC mutants}

The crystal structures of AarA ${ }^{7}$ and AarCH $6^{24}$ show the positions and likely structural consequences of the mutations identified in $A$. aceti strains 10-8 and AS10. The AarA-A265V and AarA-E92K mutations are located at the subunit interface of the core dimeric unit in positions that are far-removed from the active site (Figure 7A/B). Ala265 and Ala265' (Ala265 from a partner subunit) are buried near each other and a pseudo-twofold symmetry axis (Figure 7D). A larger valine side chain at this position may wedge apart helices $\alpha \mathrm{M}$ and $\alpha \mathrm{M}^{\prime}$. Glu92 forms a solvent-accessible salt bridge with Arg436' (dotted line in Figure 7E) that would be disrupted by a positively charged lysine side chain. The AarCH6-C357Y mutation is located near but not in the active site (Figure 7C). Cys357 is buried within a cluster of highly ordered hydrophobic residues (Figure 7F). A considerably larger tyrosine side chain would disrupt hydrophobic packing interactions and likely hinder formation of the core $\beta$-sheet in the $\mathrm{C}$-terminal domain. Nearby chloride binding sites located at the subunit interface (Figure 7C) may also be perturbed.

AarCH6-C357Y was heterologously overproduced in E. coli
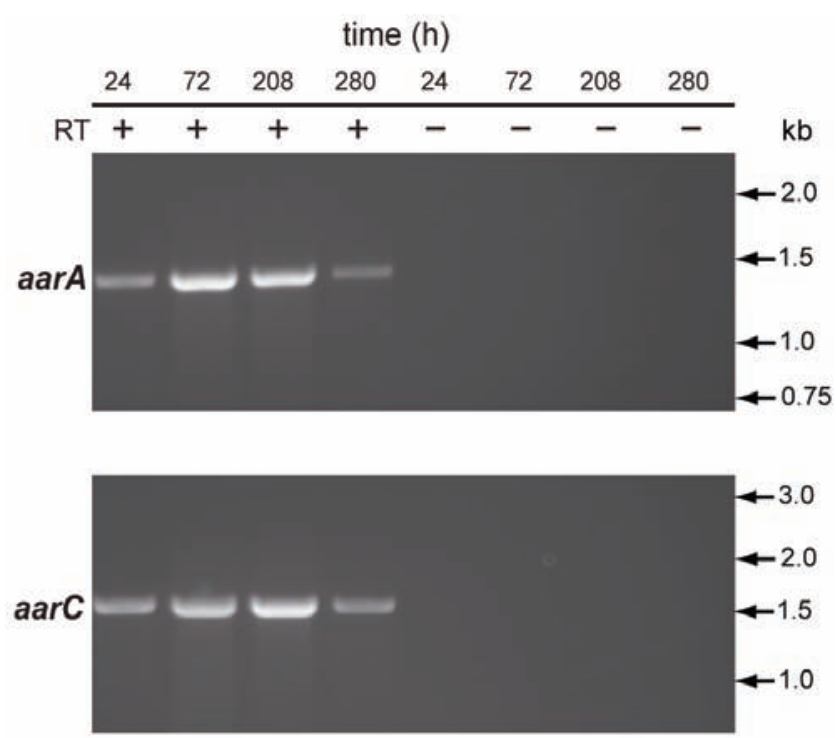

Figure 5. Analysis of aarA and $\operatorname{aar} C$ transcripts by reverse transcription-polymerase chain reaction. Size standard positions are indicated. 
C41(DE3). While AarCH6-C357Y was abundant in the total lysate, no soluble protein remained after solids were removed by centrifugation (data not shown). Furthermore, no SCACT activity was detected in recombinant $E$. coli $\mathrm{C} 41$ (DE3) lysate. Profound insolubility of AarCH6C357Y would account for the lack of SCACT activity in the lysate of recombinant $E$. coli $\mathrm{C} 41$ (DE3) and the $\mathrm{Ace}^{\mathrm{S}}$ phenotype of $A$. aceti strain AS10.

\section{Discussion}

Favored vinegar production strains accumulate and tolerate high concentrations of acetic acid, while minimizing overoxidation of acetate to carbon dioxide. ${ }^{2}$ This study examined the relationship between acetate metabolism and the functional expression of aar genes in the industrial vinegar strain A. aceti 1023. In agreement with previous reports, ${ }^{10,11}$ strain 1023 rapidly produced acetic acid in the first two days of growth as cell density increased and then maintained and tolerated a high concentration of acetic acid ( $280 \mathrm{mM}$ from 340 $\mathrm{mM}$ ethanol) for six days as cell density remained constant. On the eighth day, acetic acid depletion began as cell density once again increased. AarA and AarC were abundant during both log phases (Figure 3C,E), indicating the diauxic shift was not a simple consequence of CAC enzyme induction at the onset of the second log phase.

\section{Functional production of AarA and AarC}

CAC enzyme levels are correlated with acetic acid production and resistance.$^{25} \mathrm{~A}$. aceti strain 1023 (and its derivatives) requires AarA and AarC for both CAC flux and acetic acid resistance on solid medium. ${ }^{6}, 26$ Transcription of aarA and $a \operatorname{arC}$ was highest during the second $\log$ phase (Figure 3D), consistent with a role for the variant CAC in dissimilatory acetate metabolism. ${ }^{8}$ mRNA abundance correlated well with functional production of AarA and AarC. Based upon maximal enzyme specific activities, ${ }^{7,8}$ AarA and AarC represented about $2 \%$ and $1 \%$, respectively, of the soluble proteins present during the second log phase, about twice the level reached during the first log phase.

YPDE growth curves for $a a r C^{+}$and $a a r C^{-}$strains demonstrate that AarC is not required for ethanol oxidation, but is required for acetate overoxidation (Figure 3A,B). The first finding is consistent with the expectation that ethanol oxidation serves as the main energy source during the first log phase, while the CAC plays a supporting or
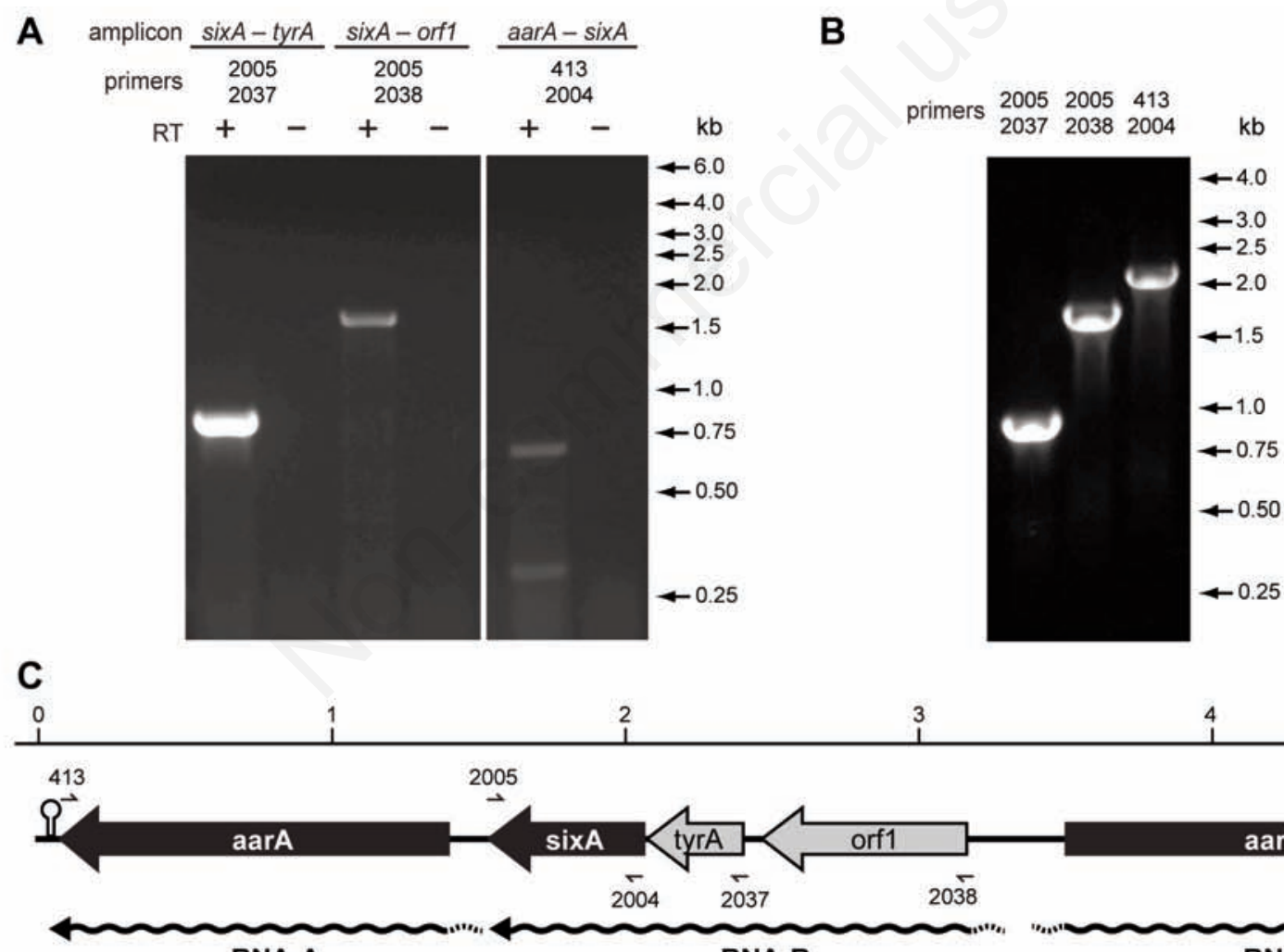

RNA-A

Figure 6. Mapping of transcripts produced from the aar gene cluster of $A$. aceti strain 1023. (A) Amplification of intergenic regions by reverse transcription-polymerase chain reaction (RT-PCR). Oligodeoxynucleotide primers were selected that would produce amplicons spanning $\operatorname{six} A$ - tyrA, 833 bp expected; sixA - orf1, 1639 bp expected; and aarA - six $A, 2049$ bp expected. The faint bands present in the $\operatorname{aar} A-\operatorname{six} A$ lane correspond to significantly shorter products than expected for the aar $A-\operatorname{six} A$ amplicon. Intermediate lanes are omitted. Size standard positions are indicated. (B) Corresponding PCR controls containing gDNA template. Size standard positions are indicated. (C) Transcripts produced by the aar gene cluster. Five proteins are encoded on three transcripts (wavy lines with 5 ' start sites shown as dotted lines). The polycistronic transcript RNA-B includes $\operatorname{six} A, \operatorname{tyr} A$, and orf1. The monocistronic transcripts RNA-A and RNA-C include a arA and aar $C$, respectively. Putative rho-independent terminator sequences (stem-loops) downstream of aarA and $a a r C$ define the aar gene cluster. Small arrows indicate the positions and polarities of ODN primers (Table 2). 
anaplerotic role. The second finding confirms that the aar genes play a role in diauxic growth in liquid medium. These data also demonstrate that $A$. aceti strain 1023 (and its derivatives) lacks a functional alternative to AarC, which confirms and extends a finding that none of the proteins produced by the other four CoA-transferase genes identified in the genome of $A$. aceti strain $1023^{23}$ has significant in vitro SCACT activity. ${ }^{8}$ Further study will be needed to determine if the new aarA alleles identified in A. aceti strains 10-8 and AS10 (Table 3) are catalytically impaired or downregulated.

\section{Acetic acid sensitivity of $A$. aceti strain AS10}

$A$. aceti cells counteract a constant influx of acetic acid. While passive acidification of the cytoplasm ${ }^{27}$ should suppress intracellular acetate accumulation, ${ }^{28}$ an energy source is required to support active acetic acid export by a proton gradient-dependent transporter ${ }^{29}$ or the $\mathrm{ABC}$ transporter AatA, ${ }^{30}$ each of which contributes to acetic acid resistance. ${ }^{31}$ AatA overexpression has been shown to improve acetic acid yields ${ }^{30}$ under moderate acetic acid levels similar to those used in this study. (Cultures used in the vinegar industry routinely reach much higher ethanol and acetic acid levels ${ }^{5}$ ). The importance of vigorous aeration $^{32,33}$ and periplasmic alcohol dehydrogenase activity 34,35 in $A$. acet $i$ and highly acetic acid resistant $\mathrm{AAB}$ underscores the critical link between aerobic energy metabolism and acetic acid resistance.

Under the conditions examined, AarC does not appear to be required prior to the onset of acetic acid consumption (Figure 3A,B). CS and SCACT activities were also greatly reduced in the first log phase of $A$. aceti strain 10-8, possibly due to a mutation outside the aar gene cluster. CAC flux in A. aceti strain AS10 was apparently insufficient to support substantial acetate overoxidation on solid ${ }^{6}$ or in liquid medium (Figure 3B), likely resulting from SCACT deficiency due to a mutation that prevents proper folding of AarC (Figure 7C,F). The lack of a second $\log$ phase suggests that aerobic acetate overoxidation and diauxic growth are linked. We suggest that AarC is required during the second log phase for two reasons: to provide the energy needed for acetic acid resistance in otherwise depleted growth medium and to eliminate cytoplasmic acetate by dissimilation. In contrast, the assimilation function originally attributed to AarC 6,26 is unlikely to significantly contribute to acetic acid resistance. ${ }^{9}$
A

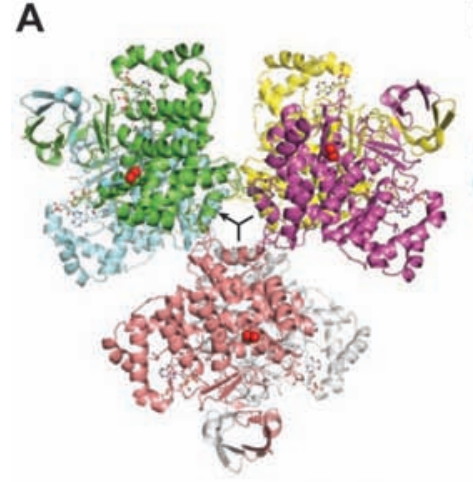

B

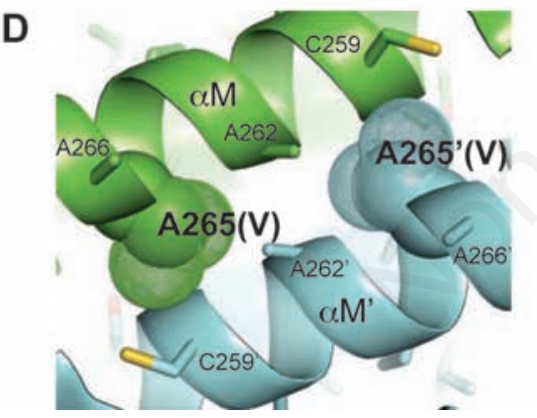

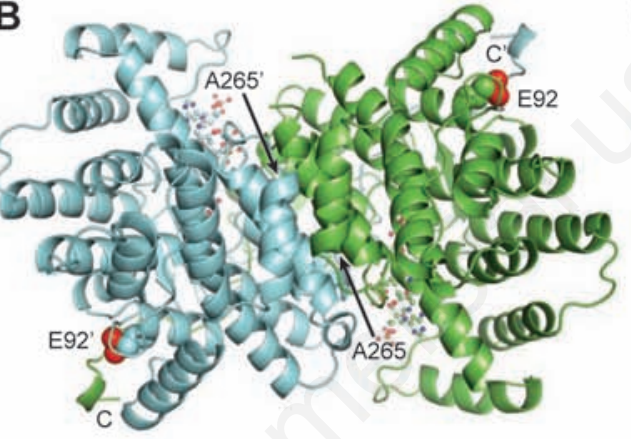

E

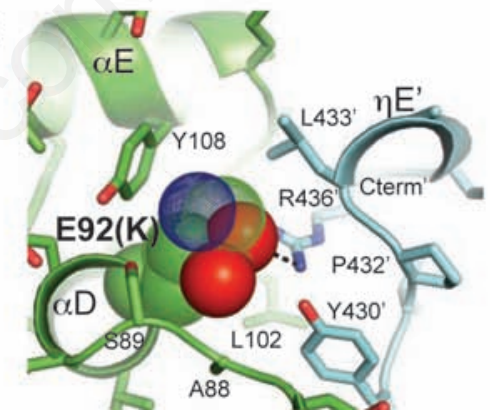

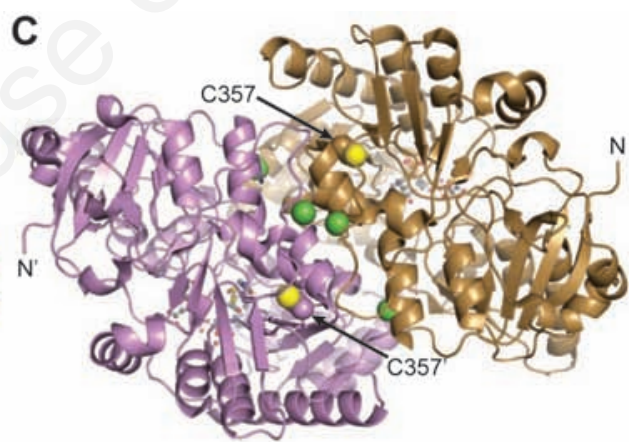

$\mathbf{F}$

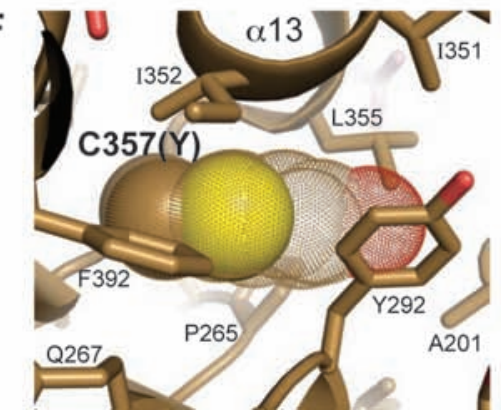

Figure 7. Modeled mutations in AarA and AarC from A. aceti strains 10-8 and AS10. In all panels, residues and secondary structural elements in subunit B are indicated with primes. (A) AarA hexamer (PDB id 2h12). The shape at the center indicates the pseudo-threefold symmetry axis. (B) AarA dimer viewed from the center of the hexamer (along the arrow at the center of panel A) showing Glu92 and Ala265 side chains rendered as spheres. Active site ligands are shown in ball-and-stick rendering; sulfate ions from the crystallization medium are omitted. Subunit $A$ is green and subunit $B$ is light blue. The $C$-terminus of each AarA subunit $\left(C\right.$ or $\left.C^{\prime}\right)$ wraps around its partner in the dimeric unit. (C) AarC dimer (PDB id 4eu7) showing Cys357 side chains rendered as spheres. Active site ligands are shown in ball-and-stick rendering; four chloride ions at the subunit interface are shown as green spheres. Subunit $\mathrm{A}$ is light brown and subunit $B$ is violet. (D) Location of the AarA-A265V mutation identified in strain 10-8. Ala265 and Ala265' (solid spheres) are situated between the pseudosymmetric helices $\alpha \mathrm{M}$ and $\alpha \mathrm{M}^{\prime}$. The Ala265 $\rightarrow$ Val substitution (dotted spheres) may wedge apart these interfacial secondary structural elements. (E) Location of the AarA-E92K mutation identified in strain AS10. A solvent-accessible salt bridge formed between Glu92 (solid spheres) and Arg436' would be disrupted by the Glu92 $\rightarrow$ Lys substitution (dotted spheres). (F) Location of the AarCC357Y mutation identified in strain AS10. Cys357 (solid spheres) is buried near a central $\beta$-sheet. The Cys357 $\rightarrow$ Tyr substitution (dotted spheres) would clash with several hydrophobic residues and disrupt a tightly packed core. Helix $\alpha 13$ contains four affected residues: Ile351, Ile352, Arg354 (not shown), and Leu355. Arg354 contacts two chloride ions located at the center of the subunit interface (the average $\mathrm{Cl}-\mathrm{S} \gamma$ distance is $13.6 \AA$ ). 


\section{Modulation of citric acid cycle enzyme synthesis by environmental inputs}

Genes involved in ethanol oxidation are regulated by quorum sensing in Gluconacetobacter strains ${ }^{36,37}$ and perhaps other AAB. Residual ethanol suppresses acetate overoxidation in $\mathrm{AAB}$ by an undefined mechanism. ${ }^{2,3,38}$ The role of SixA in the aar system may be to regulate $\mathrm{CAC}$ gene expression in one or both log phases. The relatively low abundance of SixA (Figure 4) and its production from a separate mRNA (Figure 6) are consistent with a regulatory role.

In facultative anaerobes like $E$. coli, CAC enzyme synthesis is regulated by the Arc two-component system. ${ }^{39}$ Oxygen deficiency causes the receptor kinase $\mathrm{ArcB}$ to autophosphorylate and then transphosphorylate the response regulator ArcA, which represses several operons involved in aerobic respiration. ${ }^{39-41}$ Anaerobic repression of the $s d h-$ suc operon is particularly strong. ${ }^{42}$ The anaerobic metabolites D-lactate and acetate enhance anaerobic repression by increasing $\mathrm{ArcB}$ autophosphorylation. ${ }^{43,44}$ Under anaerobic conditions, SixA attenuates repression by dephosphorylating ArcB. ${ }^{45}$

The primary role of SixA in thoroughly aerated $A$. aceti cultures may therefore be to derepress CAC enzyme synthesis at moderate to high acetic acid levels, thereby ensuring the presence of anaplerotic pathways during both log phases and a continuous energy supply during the second log phase when other nutrients are depleted.

\section{Metabolic streamlining of industrial vinegar produc- tion strains}

$\mathrm{AAB}$ with a complete $\mathrm{CAC}$ contain genes for succinyl-CoA synthetase, SCACT, or both. ${ }^{46}$ Some Acetobacter strains, including A. aceti strain NBRC 14818, also possess a functional glyoxylate shunt. ${ }^{4,47}$ Others, including $A$. aceti strain 1023, do not. ${ }^{8,48}$ In conjunction with enzymes that interconvert acetate and acetyl-CoA, these alternatives (Figure 1) support acetate assimilation, dissimilation, or both. Vinegar production strains like $A$. aceti strain 1023 that possess streamlined central carbon metabolism provide a comparatively straightforward link between metabolic flux control and acetic acid production.

Diverse metabolic pathways would equip wild Acetobacter strains to exploit the complex nutritional environment present in fruit and other natural niches. ${ }^{1}$ For industrial strains adapted to defined ethanol medium, however, a glyoxylate shunt may be superfluous and could decrease acetic acid yields. A. aceti strain GP, an $a c e A / g l c B$ mutant of NBRC 14818 that lacks a glyoxylate shunt, furnishes a higher yield of acetic acid than the parent strain. ${ }^{49}$ Selection for high vinegar yields may have favored strains like $A$. aceti strain 1023 that have a limited ability to assimilate acetate.

\section{Conclusions}

Diauxic growth in YPDE medium is accompanied by distinct stages of acetic acid accumulation, conservation, and depletion. AarC is abundant during both acetic acid accumulation and acetic acid depletion, suggesting that modulation of AarC levels is not the method by which acetate catabolism is regulated. AarC is not required for the conversion of ethanol to acetic acid, indicating that acetic acid resistance is supported by other energy sources during the first log phase. The aar genes enable aerobic growth in otherwise depleted medium during the second log phase, consistent with a dual role for the variant CAC in energy harvesting and acetate dissimilation. We speculate that acetate overoxidation is controlled by cytoplasmic acetate levels and linked to active acetic acid export, while SixA ensures that aerobic acetate metabolism proceeds in conditions that would otherwise suppress CAC flux.

\section{References}

1. Asai T. Acetic acid bacteria. Classification and biochemical activities. Tokyo: University of Tokyo Press; 1968.

2. Saeki A, Taniguchi M, Matsushita K, et al. Microbiological aspects of acetate oxidation by acetic acid bacteria, unfavorable phenomena in vinegar fermentation. Biosci Biotechnol Biochem 1997;61:317-23.

3. Saeki A, Matsushita K, Takeno S, et al. Enzymes responsible for acetate oxidation by acetic acid bacteria. Biosci Biotechnol Biochem 1999;63:2102-9.

4. Sakurai K, Arai H, Ishii M, Igarashi Y. Transcriptome response to different carbon sources in Acetobacter aceti. Microbiology 2011;157:899-910.

5. García-García I, Santos-Dueñas IM, Jiménez-Ot C, et al. Vinegar engineering. In: Solieri L, Giudici P, eds. Vinegars of the world. 1st ed. New York: Springer; 2008. pp 97-120.

6. Fukaya M, Takemura H, Okumura H, et al. Cloning of genes responsible for acetic acid resistance in Acetobacter aceti. J Bacteriol 1990;172:2096-104.

7. Francois JA, Starks CM, Sivanuntakorn S, et al. Structure of a NADH-insensitive hexameric citrate synthase that resists acid inactivation. Biochemistry. 2006;45:13487-99.

8. Mullins EA, Francois JA, Kappock TJ. A specialized citric acid cycle requiring succinyl-coenzyme A $(\mathrm{CoA})$ :acetate CoA-transferase (AarC) confers acetic acid resistance on the acidophile Acetobacter aceti. J Bacteriol 2008;190:4933-40.

9. Steiner P, Sauer U. Proteins induced during adaptation of Acetobacter aceti to high acetate concentrations. Appl Environ Microbiol 2001;67:5474-81.

10. Ohmori S, Uozumi T, Beppu T. Loss of acetic acid resistance and ethanol oxidizing ability in an Acetobacter strain. Agric Biol Chem 1982;46:381-9.

11. Ohmori S, Masai H, Arima K, Beppu T. Isolation and identification of acetic acid bacteria for submerged acetic acid fermentation at high temperature. Agric Biol Chem 1980;44:2901-6.

12. Miroux B, Walker JE. Over-production of proteins in Escherichia coli: Mutant hosts that allow synthesis of some membrane proteins and globular proteins at high levels. J Mol Biol 1996;260:289-98.

13. Bradford MM. A rapid and sensitive method for the quantitation of microgram quantities of protein utilizing the principle of proteindye binding. Anal Biochem 1976;72:248-54.

14. Ewing B, Green P. Base-calling of automated sequencer traces using Phred. II. Error probabilities. Genome Res 1998;8:186-94.

15. Ewing B, Hillier L, Wendl MC, Green P. Base-calling of automated sequencer traces using Phred. I. Accuracy assessment. Genome Res 1998;8:175-85.

16. Gordon D, Abajian C, Green P. Consed: A graphical tool for sequence finishing. Genome Res 1998;8:195-202.

17. Naville M, Ghuillot-Gaudeffroy A, Marchais A, Gautheret D. ARNold: a web tool for the prediction of Rho-independent transcription terminators. RNA Biol 2011;8:11-3.

18. Chinnawirotpisan P, Theeragool G, Limtong $S$, et al. Quinoprotein alcohol dehydrogenase is involved in catabolic acetate production, while NAD-dependent alcohol dehydrogenase in ethanol assimilation in Acetobacter pasteurianus SKU1108. J Biosci Bioeng 2003;96:564-71.

19. Ausubel FM, Brent R, Kingston RE, et al. Short protocols in molecular biology: A compendium of methods from Current protocols in molecular biology. 4th ed. New York, NY: Wiley; 1999.

20. Schneider CA, Rasband WS, Eliceiri KW. NIH Image to ImageJ: 25 years of image analysis. Nat Meth 2012;9:671-5.

21. DeLano WL. The PyMOL molecular graphics system. Palo Alto, CA: 
DeLano Scientific; 2002.

22. Grossman TH, Kawasaki ES, Punreddy SR, Osburne MS. Spontaneous cAMP-dependent derepression of gene expression in stationary phase plays a role in recombinant expression instability. Gene 1998;209:95-103.

23. Francois JA, Kappock TJ. Alanine racemase from the acidophile Acetobacter aceti. Protein Expression Purif 2007;51:39-48.

24. Mullins EA, Kappock TJ. Crystal structures of Acetobacter aceti succinyl-coenzyme A (CoA):acetate CoA-transferase (AarC) reveal specificity determinants and illustrate the mechanism used by class I CoA-transferases. Biochemistry 2012;51:8422-34.

25. Nakano S, Fukaya M, Horinouchi S. Enhanced expression of aconitase raises acetic acid resistance in Acetobacter aceti. FEMS Microbiol Lett 2004;235:315-22.

26. Fukaya $\mathrm{M}$, Takemura $\mathrm{H}$, Tayama $\mathrm{K}$, et al. The $\operatorname{ar} C$ gene responsible for acetic acid assimilation confers acetic acid resistance on Acetobacter aceti. J Ferment Bioeng 1993;76:270-6.

27. Menzel U, Gottschalk G. The internal $\mathrm{pH}$ of Acetobacterium wieringae and Acetobacter aceti during growth and production of acetic acid. Arch Microbiol 1985;143:47-51.

28. Russell JB. Another explanation for the toxicity of fermentation acids at low $\mathrm{pH}$ : anion accumulation versus uncoupling. J Appl Microbiol 1992;73:363-70.

29. Matsushita K, Inoue T, Adachi 0, Toyama H. Acetobacter aceti possesses a proton motive force-dependent efflux system for acetic acid. J Bacteriol 2005;187:4346-52.

30. Nakano S, Fukaya M, Horinouchi S. Putative ABC transporter responsible for acetic acid resistance in Acetobacter aceti. Appl Environ Microbiol 2006;72:497-505.

31. Nakano S, Fukaya M. Analysis of proteins responsive to acetic acid in Acetobacter: Molecular mechanisms conferring acetic acid resistance in acetic acid bacteria. Int J Food Microbiol 2008;125:549.

32. Muraoka H, Watabe Y, Ogasawara N. Effect of oxygen deficiency on acid production and morphology of bacterial cells in submerged acetic fermentation by Acetobacter aceti. J Ferment Technol 1982;60:171-80.

33. Park YS, Ohtake H, Fukaya M, et al. Effects of dissolved oxygen and acetic acid concentrations on acetic acid production in continuous culture of Acetobacter aceti. J Ferment Bioeng 1989;68:96-101.

34. Trcek J, Toyama H, Czuba J, et al. Correlation between acetic acid resistance and characteristics of $\mathrm{PQQ}$-dependent $\mathrm{ADH}$ in acetic acid bacteria. Appl Microbiol Biotechnol 2006;70:366-73.

35. Trcek J, Jernejc K, Matsushita K. The highly tolerant acetic acid bacterium Gluconacetobacter europaeus adapts to the presence of acetic acid by changes in lipid composition, morphological properties and PQQ-dependent ADH expression. Extremophiles
2007;11:627-35.

36. Iida A, Ohnishi Y, Horinouchi S. Control of acetic acid fermentation by quorum sensing via $N$-acylhomoserine lactones in Gluconacetobacter intermedius. J Bacteriol 2008;190:2546-55.

37. Iida A, Ohnishi Y, Horinouchi S. Identification and characterization of target genes of the Gin//GinR quorum-sensing system in Gluconacetobacter intermedius. Microbiology 2009;155:3021-32.

38. Kornmann H, Duboc P, Niederberger P, et al. Influence of residual ethanol concentration on the growth of Gluconacetobacter xylinus I 2281. Appl Microbiol Biotechnol 2003;62:168-73.

39. Iuchi S, Lin ECC. $\operatorname{arcA}(d y e)$, a global regulatory gene in Escherichia coli mediating repression of enzymes in aerobic pathways. Proc Natl Acad Sci U S A 1988;85:1888-92.

40. Iuchi S, Matsuda Z, Fujiwara T, Lin ECC. The $\operatorname{arcB}$ gene of Escherichia coli encodes a sensor-regulator protein for anaerobic repression of the arc modulon. Mol Microbiol 1990;4:715-27.

41. Gunsalus RP, Park SJ. Aerobic-anaerobic gene regulation in Escherichia coli: control by the ArcAB and Fnr regulons. Res Microbiol 1994;145:437-50.

42. Park SJ, Chao G, Gunsalus RP. Aerobic regulation of the sucABCD genes of Escherichia coli, which encode $\alpha$-ketoglutarate dehydrogenase and succinyl coenzyme A synthetase: Roles of ArcA, Fnr, and the upstream $s d h C D A B$ promoter. J Bacteriol 1997;179:413842.

43. Georgellis D, Kwon 0, Lin ECC. Amplification of signaling activity of the Arc two-component system of Escherichia coli by anaerobic metabolites. An in vitro study with different protein modules. J Biol Chem 1999;274:35950-4.

44. Rodriguez C, Kwon 0, Georgellis D. Effect of D-lactate on the physiological activity of the ArcB sensor kinase in Escherichia coli. J Bacteriol 2004;186:2085-90.

45. Matsubara M, Mizuno T. The SixA phospho-histidine phosphatase modulates the ArcB phosphorelay signal transduction in Escherichia coli. FEBS Lett 2000;470:118-24.

46. Matsutani M, Hirakawa H, Yakushi T, Matsushita K. Genome-wide phylogenetic analysis of Gluconobacter, Acetobacter, and Gluconacetobacter. FEMS Microbiol Lett 2011;315:122-8.

47. Stouthamer AH, van Boom JH, Bastiaanse AJ. Metabolism of $\mathrm{C}_{2}$ compounds in Acetobacter aceti. Antonie Van Leeuwenhoek 1963;29:393-406.

48. Azuma Y, Hosoyama A, Matsutani M, et al. Whole-genome analyses reveal genetic instability of Acetobacter pasteurianus. Nucleic Acids Res 2009;37:5768-83.

49. Sakurai K, Yamazaki S, Ishii M, et al. Role of the glyoxylate pathway in acetic acid production by Acetobacter aceti. J Biosci Bioeng 2013;115:32-6. 